\title{
Identification of the Inertial Parameters of a Humanoid Robot Using Grounded Sole Link
}

\author{
Toru Iwasaki, Gentiane Venture \\ Department of Mechanical Systems Engineering \\ Tokyo University of Agriculture and Technology \\ 2-24-16 Nakacho Koganei, Tokyo, Japan \\ venture@cc.tuat.ac.jp
}

\begin{abstract}
This paper describes the dynamics identification of humanoid robots. It is important to know correctly dynamics parameters of link and joint which constitute a robot for its control. Here, we identify the inertial parameters of legged systems using the base-link dynamics. This method generally set the base-link at the torso where gyroscope and accelerometer are installed in order to calculate base-link velocity and acceleration. We propose a technique to identify without using these sensors. This technique sets the base-link at the sole of the foot and uses the kinematic constraint of the leg connected to the ground. Thus the base-link velocity and acceleration are set to zero. Therefore we can identify without inertial sensor's noise. In this paper, we apply this technique to the humanoid robot HRP-2. First, we calculate the model and then we identify HRP-2 dynamics parameters with simulation environment.
\end{abstract}

\section{Keywords- Humanoid, Dynamics identification}

\section{INTRODUCTION}

It is important to know correctly robot's parameters for its control. For example, Computed torque method is usually used to control a robot and this method require robot model with precision. However modeling of robot with CAD data usually includes undesirable error such as wiring material. Then it is required to identify these parameters with robot motion. The classic identification of the inertial parameters of robots uses the joint torques, or their estimation [1]. However this method is affected by factors such as joint friction, elasticity and viscosity disturbance [2]. Therefore, the baselink identification method presented in [3], [4], [5], [6], [7] which does not require the torque measurements is useful to identify the dynamics of complex systems, in particular humanoid robots. The equation of motion of the robot is composed of the equation of base-link and that of joint links. The base-link equation does not include the joint torque. In this method, we can identify the robot's parameters without using the joint torque. Theoretically, we can set any link as the base-link. In general, we choose the link equipped with inertial measurement sensors such as an accelerometer and a gyro sensor. This method requires joint angles, base-link position and orientation, as well as their first and second de-

\author{
Eiichi Yoshida \\ CNRS-AIST, JRL (Joint Robotics Laboratory), \\ UMI 3218/CRT, Intelligent Systems Research Institute, \\ AIST Central 2, Umezono 1-1-1, \\ Tsukuba, Ibaraki 305-8568 Japan \\ e.yoshida@aist.go.jp
}

rivatives. In this case, these values are obtained from the inertial measurement sensors. However, these values must be filtered to obtain all the necessary information because it contains drift and other noise. The property of this filtering, like cut-off frequency, greatly affects the identification results. To our knowledge, however, this problem of noise sensitivity of the base-link method has never been addressed for humanoid robots that have no fixed base unlike industrial robot arms [8].

In this paper, in order to avoid unfavorable effects of noise filtering, we propose a new method that sets the base-link at the sole of the robot which is connected to ground. Since the sole is fixed to the ground, its velocity and accelerations known are equal to 0 . This allows us to identify the robot parameters without using inertial sensors. In this paper, we apply this method to the humanoid robot HRP-2 in virtual environment. One of the feet is used as the base-link to identify the inertial parameters from such motions as walking and dancing. The identified parameters are then validated by reconstructing the generalized force of the base-link.

\section{IDENTIFICATION METHOD}

The equation of motion of the robot is composed of matrix of link mass, tensor of inertia and center of mass and joint angles, velocity and acceleration. From the robot motion equation of the identification model is written as Eq.1 [9]

$$
\left[\begin{array}{l}
\mathrm{Y}_{\mathrm{B}} \\
\mathrm{Y}_{\mathrm{J}}
\end{array}\right] \phi=\left[\begin{array}{l}
0 \\
\tau
\end{array}\right]+\sum_{k=1}^{\mathrm{N}_{\mathrm{c}}}\left[\begin{array}{c}
\mathrm{K}_{\mathrm{Bk}} \\
\mathrm{K}_{\mathrm{Jk}}
\end{array}\right] F_{k}
$$

where:

$n: \quad$ Number of links.

$\mathrm{N}_{\mathrm{p}}$ : Number of inertial parameters of robot.

$\mathrm{Y}_{\mathrm{B}} \quad$ Observation matrix of robot motion. This ma-

$\mathrm{Y}_{\mathrm{J}}$ : trix is composed of joint angle, velocity and acceleration and transfer inertial parameters such as mass, tensor of inertia and center of 
mass to base-link forces and torques. $\mathrm{Y}_{\mathrm{B}}$ is a $6 \times N_{p}$ matrix. This matrix translates inertial parameters to base-link generalized forces. $\mathrm{Y}_{\mathrm{J}}$ is a $\mathrm{n} \times \mathrm{N}_{\mathrm{p}}$ matrix. This matrix translates inertial parameters to joint torques.

$\phi: \quad$ All inertial parameters of the robot. This vector contains all link mass, tensor of inertia and center of mass. This vector denoted as

$$
\begin{aligned}
& \phi=\left[\begin{array}{lll}
\phi_{1} & \cdots & \phi_{n}
\end{array}\right]^{\mathrm{T}} \\
& \phi_{i}=\left[m_{i} m s_{x i} m s_{y i} m s_{z i}\right. \\
& \left.I_{x x i} I_{y y i} I_{z z i} I_{x y i} I_{y z i} I_{z x i}\right]
\end{aligned}
$$

where:

$m_{i}[\mathrm{~kg}]$ is the mass of link i.

$m s_{x i}, m s_{y i}, m s_{z i}[\mathrm{~kg} \cdot \mathrm{m}]$ are the first moment of inertia of link i.

$I_{x x i}, I_{y y i}, I_{z z i}, I_{x y i}, I_{y z i}, I_{z x i}\left[\mathrm{~kg} \cdot \mathrm{m}^{2}\right] \quad$ are the tensor of inertial of link $i$.

$\tau$ : Joint torque vector. $\tau$ is a $\mathrm{n} \times 1$ vector.

$\mathrm{N}_{\mathrm{c}}$ : Number of contact point.

$\mathrm{K}_{\mathrm{Bk}}$ Transfer matrix of contact force to forces and $\mathrm{K}_{\mathrm{Jk}}$ : torques. $\mathrm{K}_{\mathrm{Bk}}$ is a $6 \times 6$ matrix. This matrix translates contact forces and torques to force applied to the base-link. $\mathrm{K}_{\mathrm{Jk}}$ is a $\mathrm{n} \times 6$ matrix. This matrix translates contact forces and torques to torques applied to the links.

$F_{k}$ : $\quad$ Contact force of robot and environment. $F_{k}$ is a $6 \times 1$ vector.

Eq. 1 is the full motion equation of robot but includes inertial parameters that have no direct effect on the motion. As it is preferable to have a minimal set of inertial parameters for identification [5], [6], [7], we wrote the identification model for minimal identification as follows.

$$
\left[\begin{array}{c}
\mathrm{Y}_{\mathrm{BB}} \\
\mathrm{Y}_{\mathrm{JB}}
\end{array}\right] \phi_{B}=\left[\begin{array}{l}
0 \\
\tau
\end{array}\right]+\sum_{k=1}^{\mathrm{N}_{\mathrm{c}}}\left[\begin{array}{c}
\mathrm{K}_{\mathrm{Bk}} \\
\mathrm{K}_{\mathrm{Jk}}
\end{array}\right] F_{k}
$$

where:

$\mathrm{N}_{\mathrm{B}}: \quad \mathrm{N}_{\mathrm{B}}$ is a number of base inertial parameters.

$\mathrm{Y}_{\mathrm{BB}} \quad$ Observation matrix of robot motion. These ma-

$\mathrm{Y}_{\mathrm{JB}}$ : trixes are calculated from $\mathrm{Y}_{\mathrm{B}}$ and $\mathrm{Y}_{\mathrm{J}}$ by minimize method. $Y_{B B}$ is a $6 \times N_{B}$ matrix. This matrix translates base inertial parameters to base-link forces and torques. $\mathrm{Y}_{\mathrm{J}}$ is a $\mathrm{n} \times \mathrm{N}_{\mathrm{B}}$ matrix. This matrix translates base inertial parameters to joint torques.

$\phi_{\mathrm{B}}$ : $\quad$ Base inertial parameters of the robot. This vector contains minimized inertial parameters, which calculated from $\phi . \phi_{\mathrm{B}}$ is a $\mathrm{N}_{\mathrm{B}} \times 1$ matrix.
Eq. 2 consists of the base-link equation and the joint equation. The upper part of Eq. 2 is the base-link equation that is calculated by only angle, velocity, acceleration of joint and contact forces. Thus, humanoid robot's parameters are identified with Eq. 3.

$$
\mathrm{Y}_{\mathrm{BB}} \phi_{B}=\sum_{k=1}^{\mathrm{N}_{\mathrm{c}}} \mathrm{K}_{\mathrm{Bk}} F_{k}
$$

In addition, $\mathrm{Y}_{\mathrm{BB}}$ and $\phi_{B}$ depend on the excitation of robot motion. We use a method of numerical computation to get the minimal model [10], [11], [12], [13]. This equation has no joint torques. Therefore, this method is not affected by joint disturbance factors such as joint friction, elasticity and viscosity disturbance. In this method, any link can be selected as the base-link. It is usually the link that has inertial measurement sensors. The observation matrix in Eq. 3 is calculated from the value that is obtained from these sensors that are prone to such noises as drift. Consequently, identification using inertial measurement sensors is greatly affected by the accuracy of these sensors. In case of humanoid robots, such sensors as accelerometer or gyro sensors are generally fixed at the torso link. In order to eliminate undesirable influences from the noise of those sensors, we propose a method that uses one of the soles as the base-link. The advantage of this choice is that the inertial parameters can be identified without sensors since the velocity and the acceleration of the base-link is zero. This method can be used only when the foot is the fully attached to the ground. Therefore, it is necessary to know the contact state of the robot and environment to identify the inertial parameters with the proposed method.

\section{Simulation}

\section{A. Identification model}

The proposed method is tested on a humanoid robot HRP2. This robot has a total of 30 rotational degrees of freedom. In this simulation, we set the base-link position to the right sole of HRP-2 that has the contact to floor surface in our method (We can set it similarly to the left). This configuration is like an identification way for industrial robot [3]. Fig. 1 shows these configurations. We write the identification model with this configuration.

\section{B. Motion for the identification}

We use three types of motion for the identification. One is walk forward motion (walk1) [14], [15], the second one is walk sideways (walk2) and the third is dance motion (dance) [16]. Fig.2 and fig.3 shows the scene of these motions. The robot's variables: joint angle, gyro sensor, acceleration sensor, force sensor values are sampled every $5 \mathrm{~ms}$ in the virtual environment. Joint angles and gyro, acceleration 
sensor's values are filtered by butterworth filter. This filter is configured as low pass filter with $20 \mathrm{~Hz}$ cut-off frequency.

In this simulation, we use only data when the sole of the right leg is grounded. In this identification method, we need to consider the contact state of the robot and the surrounding environment. Fig. 4 shows the value of right ankle force sensor of HRP-2 in motion of walk1. We assume that robot contact with only one side of sole when vertical contact force $F_{z}$ is close to its own weight. Then we extract the data set of the robot state. Fig.5 shows the extracted force $F_{z}$.

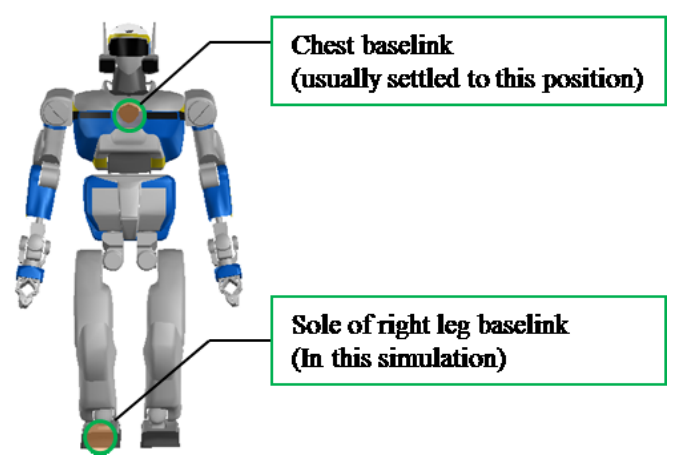

Fig.1 Configuration of base-link position

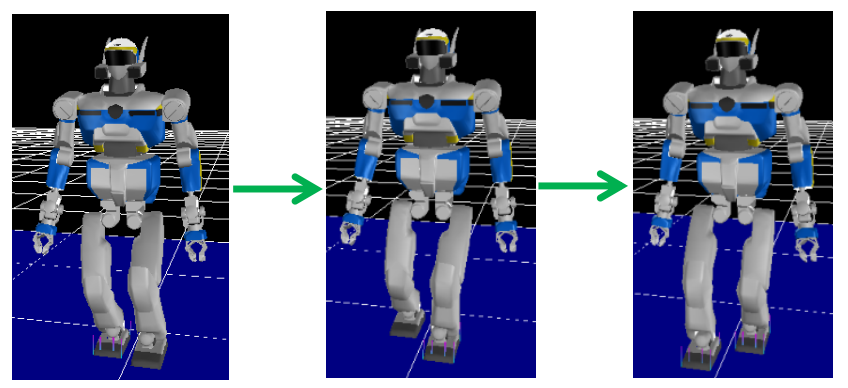

Fig.2 Motion for the identification (walk1)

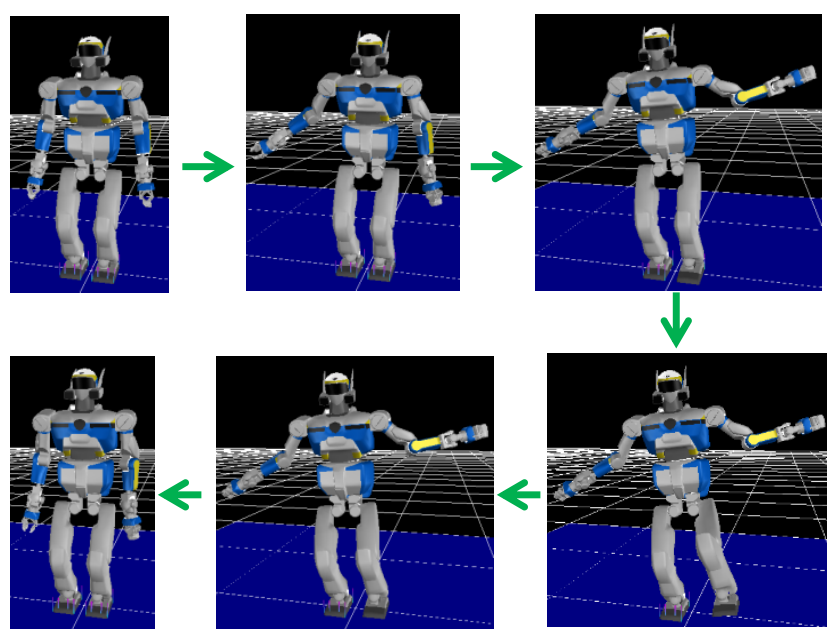

Fig.3 Motion for the identification (dance)

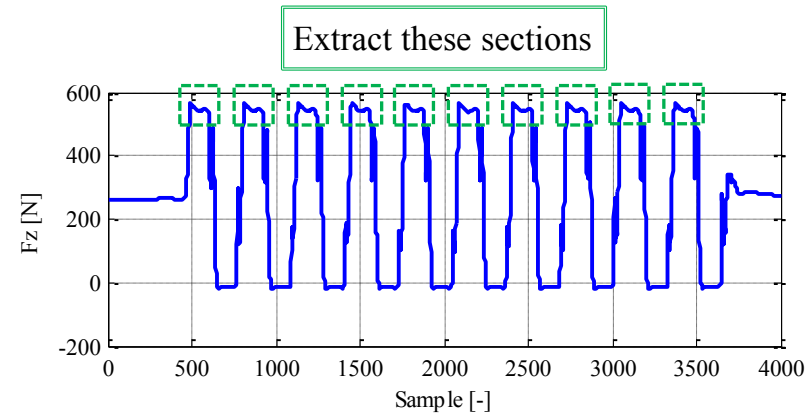

Fig.4 Raw value of vertical right ankle force sensor

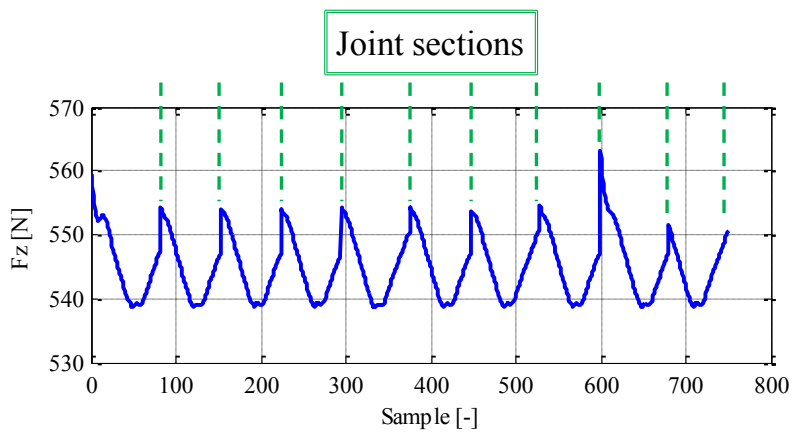

Fig.5 Extracted value of right ankle vertical force sensor when the right foot is fully grounded.

\section{SIMULATION RESULT}

\section{A. Identification and reconstruction of force}

We identify the inertial parameters by using the identification method described above for each robot motion. We compare the data obtained from the sensors modeled in the simulation and the reconstructed force applied to the right ankle to validate the correctness of the identified parameters The reconstructed base-link force is calculated by Eq.4. This calculation is called direct validation when observation matrix is the same as used for identification.

$$
F_{r c}=\mathrm{Y}_{\mathrm{id}} \phi_{i d}
$$

where:

$\mathrm{Y}_{\text {id }}$ : Minimized observation matrix

$\phi_{i d}: \quad$ Identified inertial parameters

$F_{r c}: \quad$ Reconstructed base-link force

Fig. 6 shows the results of walk 1 motion and Fig. 7 shows the results of walk 2 . The generalized force was reproduced from the parameters of the sensor data acquisition and identification results are almost identical, and that can be identified well with walk1. On the other hand difference is observed in force in several reconstructions for walk2. This can be attributed to the slip behavior in the transverse direction the sole moves laterally and there is a rolling of the foot. This violates the assumption base-link velocity and acceleration is fixed to be 0 . Table 1 and 2 shows the mean and standard deviation of these differences. Table 3 shows the 
comparison of identified inertial parameters and reference CAD data of HRP-2 and relative standard deviation of the identified parameters with walk1. These parameters are regrouped by minimize method[12].

TABLE I. MEAN AND STANDARD DEVIATION OF ERROR ABOUT DIRECT VALIDATION OF WALK1

\begin{tabular}{|c|c|c|}
\hline & Mean of error & Standard deviation of error \\
\hline $\mathrm{Fx}$ & $-1.08 \times 10^{-3}$ & $3.90 \times 10^{-2}$ \\
\hline $\mathrm{Fy}$ & $1.28 \times 10^{-3}$ & $6.13 \times 10^{-2}$ \\
\hline $\mathrm{Fz}$ & 0.00 & $3.44 \times 10^{-2}$ \\
\hline $\mathrm{Nx}$ & 0.00 & $5.34 \times 10^{-2}$ \\
\hline $\mathrm{Ny}$ & 0.00 & $3.22 \times 10^{-2}$ \\
\hline $\mathrm{Nz}$ & $-3.50 \times 10^{-5}$ & $1.90 \times 10^{-2}$ \\
\hline
\end{tabular}

TABLE II. MEAN AND STANDARD DEVIATION OF ERROR ABOUT DIRECT VALIDATION OF WALK2

\begin{tabular}{|c|c|c|}
\hline & Mean of error & Standard deviation of error \\
\hline $\mathrm{Fx}$ & $-8.43 \times 10^{-3}$ & $3.35 \times 10^{-1}$ \\
\hline $\mathrm{Fy}$ & $1.13 \times 10^{-1}$ & 7.93 \\
\hline $\mathrm{Fz}$ & 0.00 & 2.23 \\
\hline $\mathrm{Nx}$ & 0.00 & 5.71 \\
\hline $\mathrm{Ny}$ & 0.00 & $4.01 \times 10^{-1}$ \\
\hline $\mathrm{Nz}$ & $5.61 \times 10^{-3}$ & $6.24 \times 10^{-1}$ \\
\hline
\end{tabular}

TABLE III. COMPARISON OF THE IDENTIFIED INERTIAL PARAMETERS WITH WALK 1

\begin{tabular}{|c|c|c|c|c|}
\hline Parameters & CAD data & Identified & RSD [\%] & Notes \\
\hline M1 & 55.38 & 55.38 & 0.07 & \multirow{3}{*}{ Right sole } \\
\hline MX1 & -4.48 & -3.65 & 2.15 & \\
\hline MY1 & 8.35 & 7.89 & 2.80 & \\
\hline MX2 & 16.06 & 16.15 & 1.12 & \multirow{5}{*}{ Right shank } \\
\hline MY2 & 6.05 & 6.23 & 3.95 & \\
\hline XZ2 & -0.47 & -0.76 & 6.48 & \\
\hline YZ2 & -1.35 & -2.43 & 9.00 & \\
\hline ZZ2 & 18.80 & 18.83 & 1.06 & \\
\hline MX3 & 12.66 & 11.63 & 1.61 & Right thigh \\
\hline MX11 & 0.12 & 0.66 & 5.99 & \multirow{3}{*}{ Trunk } \\
\hline MY11 & -2.99 & -3.52 & 2.33 & \\
\hline YZ11 & -0.17 & 0.26 & 29.29 & \\
\hline MX13 & 0.18 & -0.23 & 9.23 & \multirow{5}{*}{ Left thigh } \\
\hline MY13 & -0.82 & -0.59 & 4.83 & \\
\hline $\mathrm{XZ13}$ & -0.03 & 0.04 & 29.73 & \\
\hline YZ13 & -0.06 & -0.06 & 19.80 & \\
\hline ZZ13 & 0.33 & 0.15 & 15.42 & \\
\hline MX18 & -0.39 & -0.67 & 2.17 & \multirow{2}{*}{ Left shank } \\
\hline MY18 & -1.15 & -0.78 & 2.69 & \\
\hline MX25 & 0.19 & 0.36 & 4.98 & \multirow{2}{*}{ Left sole } \\
\hline MY25 & 0.16 & 0.03 & 41.38 & \\
\hline
\end{tabular}
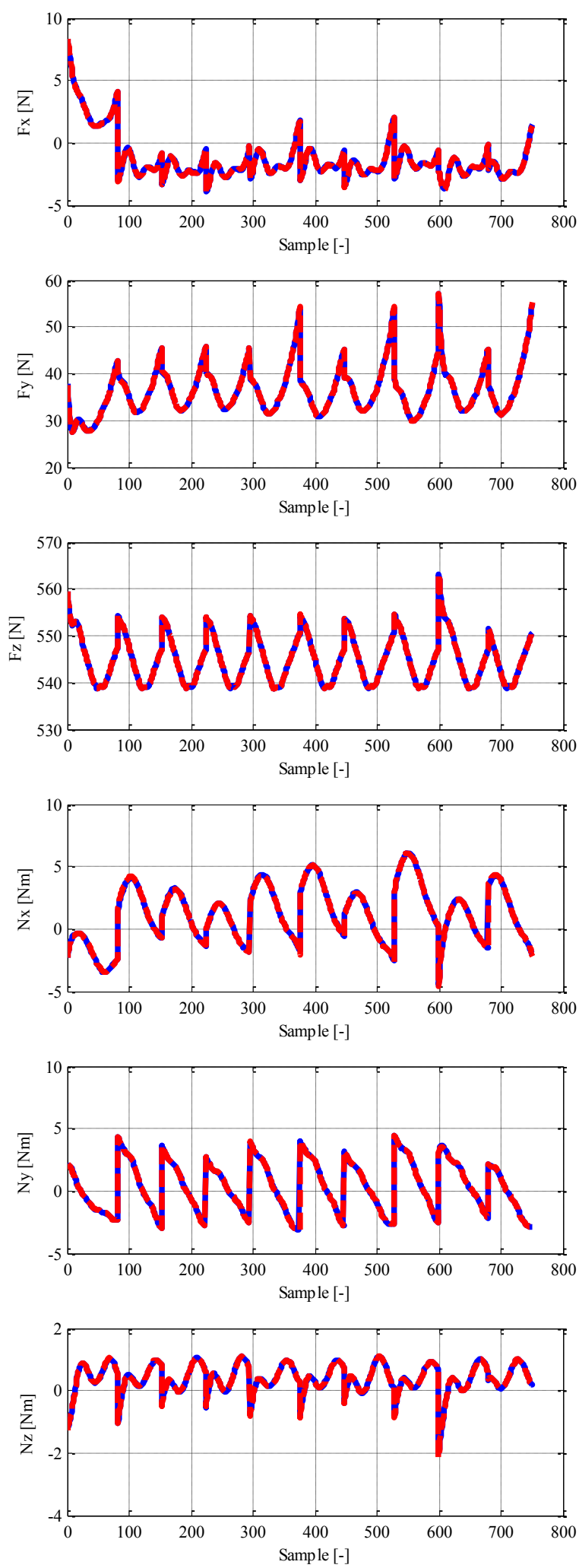

Fig.6 Comparison of sensor values and reconstructed forces. Blue line is value of force sensor. Red line is reconstructed force (Direct validation of walk1). 

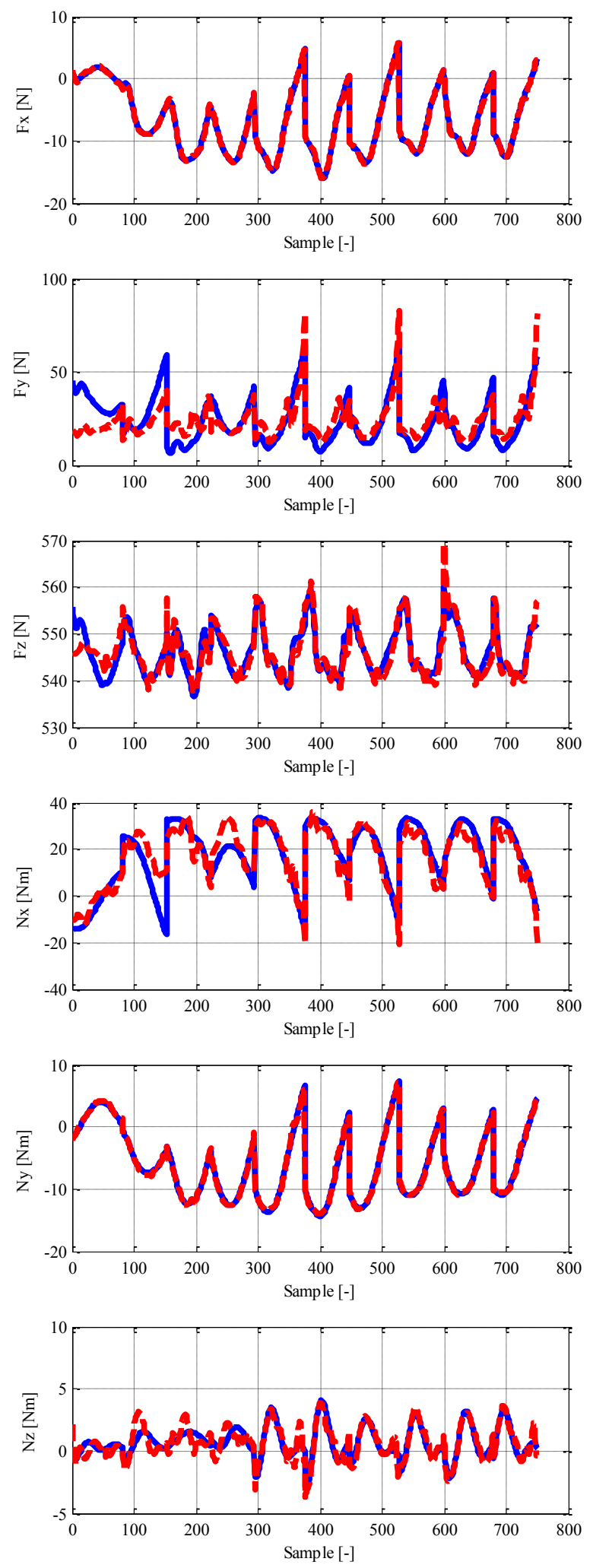

Fig.7 Comparison of sensor values and reconstructed forces. Blue line is value of force sensor. Red line is reconstructed force (Direct validation of walk2).

\section{B. Cross validation}

We carried out reconstruction of the behavior of another generalized force using the parameters identified in a certain behavior, which is called cross validation. $\mathrm{Y}_{\mathrm{BB}}$ and the generalized efforts are measured for a different movement than the one used for identification. Then we reconstruct the generalized force of dance motion from the result of walk1 identification. Fig. 8 shows the reconstructed force of dance. The generalized force has been reconstructed correctly in general, although some errors are recognized. Table 4 shows the mean and standard deviation of differences. We can therefore conclude confirm that the sole base-link identification method is effective to identify the inertial parameters of humanoid robots.

TABLE IV. MEAN AND STANDARD DEVIATION OF ERROR ABOUT CROSS VALIDATION OF DANCE

\begin{tabular}{|c|c|c|}
\hline & Mean of error & Standard deviation of error \\
\hline $\mathrm{Fx}$ & $1.46 \times 10^{-3}$ & $3.22 \times 10^{-1}$ \\
\hline $\mathrm{Fy}$ & $9.50 \times 10^{-2}$ & 1.38 \\
\hline $\mathrm{Fz}$ & $-7.63 \times 10^{-1}$ & $7.00 \times 10^{-1}$ \\
\hline $\mathrm{Nx}$ & 6.41 & 1.45 \\
\hline $\mathrm{Ny}$ & -1.74 & $6.20 \times 10^{-1}$ \\
\hline $\mathrm{Nz}$ & $-4.99 \times 10^{-3}$ & $3.95 \times 10^{-1}$ \\
\hline
\end{tabular}

\section{CONCLUSION}

In this paper, we proposed a new method to identify the inertial parameters of humanoid robot. This method is based on the equation of base-link that allows identification of robot parameters without measuring joint torque, which induces disturbances due to friction, elasticity, or viscosity. The novelty of the proposed method lies in its usage of a sole of the humanoid as the base-link by taking advantage of the zero velocity and acceleration while the foot is fixed to the ground. Using this technique, we can conduct the identification without effect of the noise of the inertial sensors such as accelerometers and gyro sensors.

We applied the proposed identification method to simulated motions of the humanoid HRP-2. We first identified the inertial parameters of the robot from the joint angle, velocity, acceleration, force sensors values the simulated robot. The simulated force sensor data are compared to the generalized force at the base-link reconstructed from the identified parameters. With two different types of walking, the reconstructed forces were generally in good accordance with the sensor data. We also observed that the identification is perturbed when the condition of zero velocity and acceleration of the sole is not satisfied due to slipping. This issue will be addressed in future work. We also apply this method to real HRP-2 and test the effectiveness of this method. 

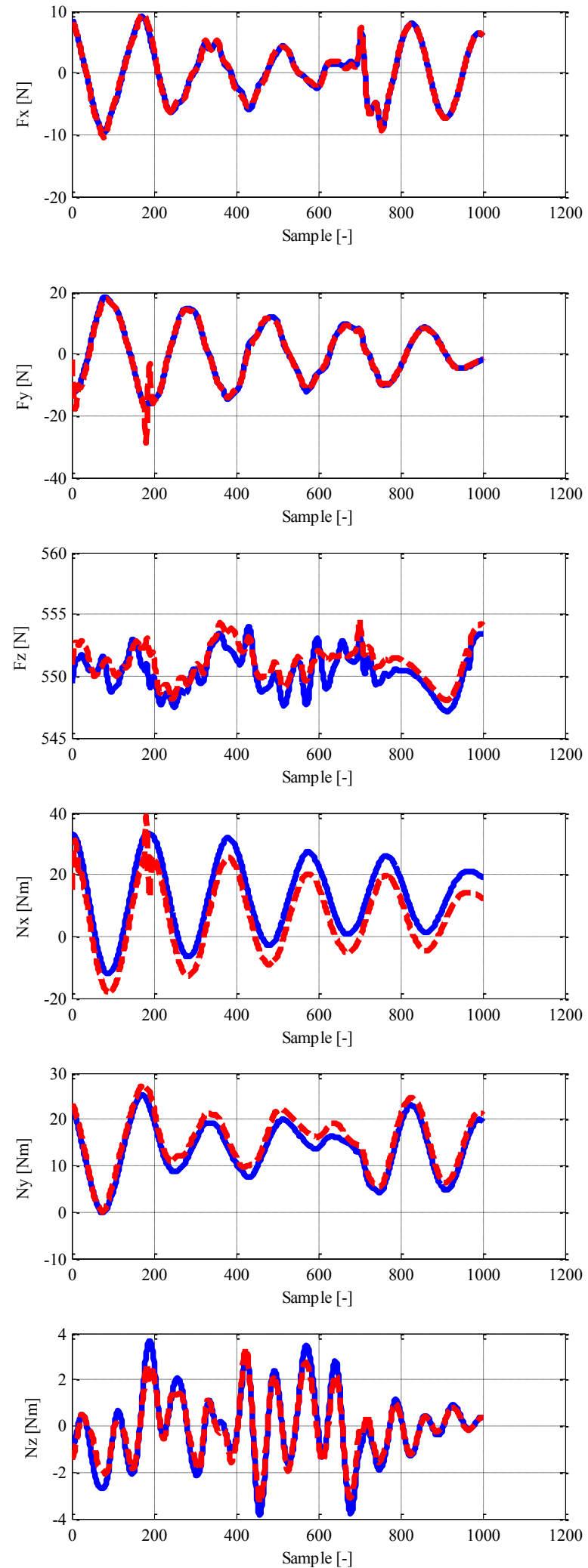

Fig. 8 Comparison of sensor values and reconstructed forces. Blue line is value of force sensor. Red line is reconstructed force. Cross validation of dance with parameters identified from walk1 motion.

\section{ACKNOWLEDGEMENT}

The authors thank Dr. A. Kheddar : CNRS-AIST, JRL (Joint Robotics Laboratory), UMI 3218/CRT, Intelligent Systems Research Institute, and Dr. O. Ramos Ponce, Dr. N. Mansard, Dr. O. Stasse : Humanoid Motion Group (Gepetto), LAAS-CNRS, Toulouse, France for their help in the data acquisition.

\section{REFERENCES}

[1] S. Bruno, K. Oussama, "Springer Handbook of Robotics", 2008

[2] F. Caccavale, P. Chlacchio, "Identification of Dynamic parameters and feedforward control for a conventional Industrial Manipulator," Control Eng. practice, Vol. 2, No. 6, pp. 1039-1050, 1994

[3] G. Liu, K. Iagnemma, S. Dubowsky and G. Morel, "A Base Force/Torque Sensor Approach to Robot Manipulator Inertial Parameter Estimation,” IEEE Int. Conf. on Robotics and Automation, pp. 3316-3321, 1998

[4] G. Venture, K. Ayusawa and Y. Nakamura, "Proprioceptive Identification of the Inertial Parameters of Humanoid Robot," 14th Robotics Symposia, pp.516-521, 2009

[5] K. Ayusawa, G. Venture and Y. Nakamura, "Identification of the Inertial Parameters of a Humanoid Robot Using Unactuated Dynamics of the Base-link," IEEE Int. Conf. on Humanoids, 2008

[6] K. Ayusawa, G. Venture, and Y. Nakamura.Identification of humanoid robots dynamics using floating-base motion dynamics. In IEEE/RSJ Int. Conf. on Intelligent Robots and Systems, pp. 2854 2859, 2008

[7] K. Ayusawa, G. Venture, and Y. Nakamura, "Minimal-set of Inertial Parameters Identification of Legged Robots Using Base-link Dynamics," Journal of Robotics Society of Japan, pp.1066-1077, 2009

[8] A. Kengo, G. Venture and Y. Tagawa, "Vibration control of an industrial robot with a flexible arm using IDCS," Proc. of Int. Conf ROMANSY, Paris, France, 2012

[9] W. Khalil, D. Creusot. "Symoro+: a system for the symbolic modelling of robots," Robotica, vol. 15, pp.153-161, 1997

[10] M. Gautier, W. Khalil, "Direct Calculation of Minimum Set of Inertial Parameters of Serial Robots," IEEE Transactions on Robotics and Automation. vol6.No3. pp. 368-373, june 1990

[11] H. Kawasaki, Y. Beniya, and K. Kanzaki, "Minimum dynamics parameters of tree structure robot models," IECON vol. 2, pp. 1100 1105,1991

[12] M. Gautier, "Numerical calculation of the base inertial parameters," J. of Robotic Systems, vol. 8(4), pp. 485-506, August 1991

[13] M. Gautier, W. Khalil, "Exciting Trajectories for the Identification of Base Inertial Parameters of Robots," Proceedings of the 30th Conference on Decision and Control Brighton, England, pp. 494-499, December 1991

[14] E. Yoshida, J.P. Laumond, "Motion planning for humanoid robots: highlights with HRP-2," Journées Nationales de la Recherche en Robotique (JNRR 2007), Obernai (France), 9-12 Octobre 2007

[15] S. Kajita, F. Kanehiro, K. Kaneko, K. Fujiwara, K. Harada, K. Yokoi and H. Hirukawa, "Biped Walking Pattern Generation by using Preview Controlof Zero-Moment Point,” Proc. 2003 IEEE Int. Conf on Robotics and Automation, pp. 1620-1626, 2003

[16] O. Ramos-Ponce, L. Saab, S. Hak, N. Mansard, "Dynamic Motion Capture and Edition using a Stack of Tasks", IEEE Int. Conf. on Humanoids, 2011 